Open Access

\title{
Primary sclerosing cholangitis associated with CREST (calcinosis, Raynaud phenomenon, oesophageal dysmotility, sclerodactyly and telangiectasia) in an elderly woman: a case report
}

\author{
Alice Powell ${ }^{1 *}$ and Julian McNeil ${ }^{2}$
}

\begin{abstract}
Introduction: CREST (calcinosis, Raynaud phenomenon, oesophageal dysmotility, sclerodactyly, and telangiectasia) syndrome comprising calcinosis cutis, Raynaud phenomenon, esophageal dysmotility, sclerodactyly and telangiectasia and primary sclerosing cholangitis are both chronic fibrotic diseases but the association between them is extremely rare. While primary sclerosing cholangitis has been associated with diffuse cutaneous scleroderma, the association with limited cutaneous scleroderma or CREST has not been previously reported in the literature. This case report illustrates the association between CREST and primary sclerosing cholangitis.

Case presentation: We report the case of an 84-year-old Asian woman with a long history of CREST who was admitted with abdominal pain, fatigue and progressive derangement of her liver enzymes. This was initially thought to be secondary to her bosentan therapy for pulmonary hypertension but it persisted despite bosentan being ceased. Primary sclerosing cholangitis was subsequently diagnosed on magnetic resonance cholangiopancreatography and she was referred to a hepatologist for treatment.

Conclusions: This case highlights the need to consider primary sclerosing cholangitis in patients with CREST who present with abdominal symptoms and deranged liver enzymes when other causes have been excluded. Relevant differential diagnoses for this presentation, which can be difficult to exclude, include immunoglobulin G4-associated cholangitis and antimitochondrial antibody negative primary biliary cirrhosis. It is of particular significance to rheumatologists and gastroenterologists but has broader relevance to all medical specialists involved in the care of patients with CREST.
\end{abstract}

Keywords: Bosentan, Cholestasis, CREST, Liver toxicity, Primary sclerosing cholangitis

\section{Introduction}

Primary sclerosing cholangitis (PSC) is a rare idiopathic chronic progressive liver disease resulting in fibroobliterative inflammation of the hepatic bile ducts and ultimately cirrhosis and liver failure [1]. It is more frequent in men and strongly associated with inflammatory bowel disease $[1,2]$. The liver disease usually associated with scleroderma is primary biliary cirrhosis $(\mathrm{PBC})$ with

\footnotetext{
* Correspondence: alice.c.powell@gmail.com

${ }^{1}$ The Queen Elizabeth Hospital, Adelaide, SA, Australia

Full list of author information is available at the end of the article
}

approximately $15 \%$ of patients with $\mathrm{PBC}$ reported to have scleroderma [3]. The association was first made by Murray-Lyon et al. in the 1970s [4]. In contrast, the relationship between PSC and scleroderma is extremely rare [3] with only one case report, to the best of our knowledge, of PSC and diffuse cutaneous scleroderma [2]. We describe a case of PSC occurring in a patient with CREST (calcinosis cutis, Raynaud phenomenon, esophageal dysmotility, sclerodactyly and telangiectasia) syndrome, which is a type of limited cutaneous scleroderma.

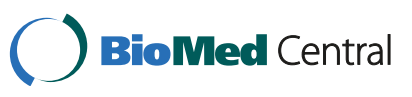

(c) 2015 Powell and McNeil. Open Access This article is distributed under the terms of the Creative Commons Attribution 4.0 International License (http://creativecommons.org/licenses/by/4.0/), which permits unrestricted use, distribution, and reproduction in any medium, provided you give appropriate credit to the original author(s) and the source, provide a link to the Creative Commons license, and indicate if changes were made. The Creative Commons Public Domain Dedication waiver (http://creativecommons.org/publicdomain/zero/1.0/) applies to the data made available in this article, unless otherwise stated. 


\section{Case presentation}

An 84-year-old Asian woman was electively admitted to our hospital for investigation of a 6-month history of liver function test (LFT) derangement, abdominal pain, fatigue and weight loss. The results of her LFTs were as follows: alkaline phosphatase $953 \mathrm{U} / \mathrm{L}$, gamma glutamyl transpeptidase $1164 \mathrm{U} / \mathrm{L}$, alanine aminotransferase $81 \mathrm{U} / \mathrm{L}$, aspartate aminotransferase $89 \mathrm{U} / \mathrm{L}$, bilirubin $13 \mu \mathrm{mol} / \mathrm{L}$ and albumin $33 \mathrm{~g} / \mathrm{L}$. She had experienced Raynaud's phenomenon for 50 years and had a confirmed diagnosis of CREST complicated by pulmonary hypertension, for which she had been treated with bosentan for the past 7 years. Her abdominal pain was initially attributed to esophageal dysmotility associated with her CREST and had improved with pantoprazole.

The most frequent severe side effect of bosentan is liver toxicity, typically manifested by a transaminasemia [5]. Of interest, animal models have demonstrated that bosentan may also cause cholestatic liver injury through inhibition of the bile salt export pump (BSEP) [6]. Our patient's LFT derangement was initially attributed to bosentan and this was decreased and then changed to macitentan which does not interfere with BSEP [6]. However, her LFT derangement gradually worsened despite these measures and therefore other causes were sought. There were no other new medications, her hepatitis serology was negative and there was no evidence of cholecystitis, cholelithiasis or biliary obstruction on ultrasound. Computed tomography $(\mathrm{CT})$ of her abdomen was also unremarkable. An autoimmune screen revealed positive antinuclear antibody consistent with CREST, antimitochondrial antibody and other liver autoantibodies negative. Immunoglobulin levels including immunoglobulin G4 (IgG4) were normal. Tumor markers including cancer antigen 19-9 (CA 19-9) and carcinoembryonic antigen (CEA) were similarly normal. She proceeded to magnetic resonance cholangiopancreatography (MRCP) which demonstrated a prominent common bile duct and prominent irregular central hepatic ducts with areas of beading and narrowing in both lobes favoring stricturing and PSC (Fig. 1).

A liver biopsy was not pursued given her age and comorbidities, in particular her pulmonary hypertension. While this may have provided useful additional information particularly in the exclusion of other differential diagnoses, findings can be nonspecific [7]. In addition, a biopsy is generally not required for the diagnosis of PSC where cholestatic biochemical abnormalities and characteristic changes on cholangiography are present.

\section{Discussion}

PSC is a complex heterogeneous probably immunemediated disease with an unpredictable clinical presentation and course. Diagnosis is made in patients with a cholestatic biochemical profile and characteristic bile

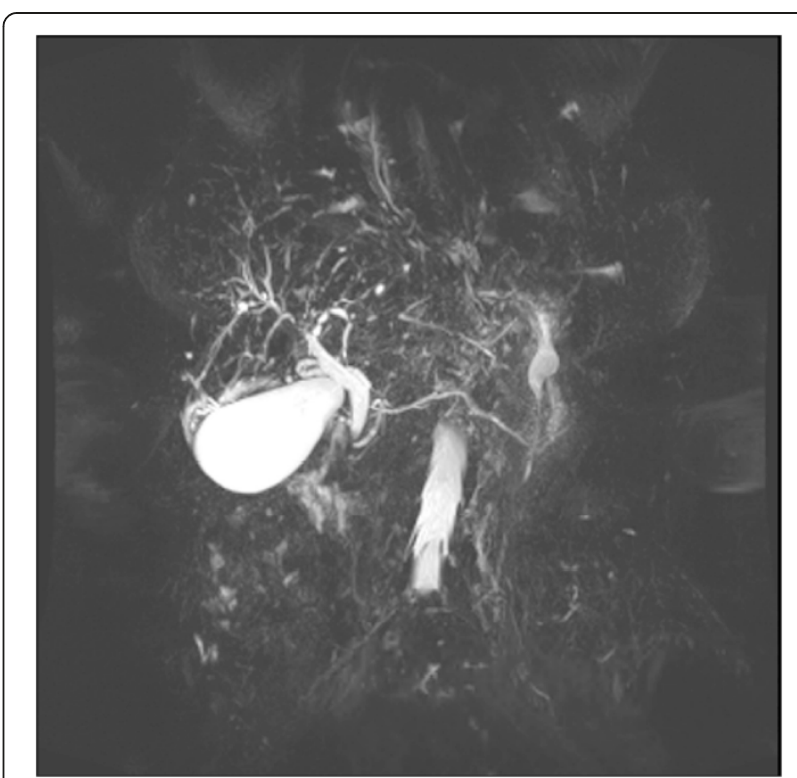

Fig. 1 Magnetic resonance cholangiopancreatography demonstrating prominent common bile duct and prominent irregular hepatic ducts

duct changes on cholangiography (MRCP or endoscopic retrograde cholangiopancreatography, ERCP) where secondary causes of sclerosing cholangitis have been excluded [1]. Typical symptoms include right upper quadrant abdominal pain, fatigue, pruritus and weight loss. Autoantibodies have no role in the routine diagnosis of PSC but antimitochondrial antibody is usually negative whereas it is positive in PBC [1]. Abdominal ultrasound and CT findings are usually nonspecific and ERCP has been regarded as the gold standard for diagnosis. However, MRCP has comparable diagnostic accuracy and is noninvasive without the potential for pancreatitis and bacterial cholangitis [1]. In a metaanalysis of six studies, the sensitivity and specificity of MRCP for diagnosing PSC were 86 and $94 \%$ respectively [8]. In the presence of an abnormal cholangiogram, a liver biopsy is not required to establish a diagnosis of PSC. No drug therapy has been conclusively proven to alter the natural history of this disorder and liver transplantation is the treatment of choice for advanced liver disease [1].

PSC should be differentiated from secondary causes of sclerosing cholangitis and IgG4-associated cholangitis. Another potential differential diagnosis for this case was antimitochondrial antibody negative $\mathrm{PBC}$ as antimitochondrial antibody may be negative in less than $10 \%$ of cases [9]. However, patients with $\mathrm{PBC}$ should have a normal biliary tree on cholangiography [9]. Secondary causes of PSC were ruled out on the basis of our patient's history, physical examination and imaging studies. Of importance, there was no dominant stricture on MRCP. IgG4-associated cholangitis is more difficult 
to differentiate and it is possible that IgG4-associated cholangitis, autoimmune pancreatitis and PSC are different manifestations of the same disease [10]. This is, however, an important distinction as IgG4-associated cholangitis may be glucocorticoid responsive [10], while no effective medical therapy is available for PSC. This diagnosis was thought less likely in our patient's case as IgG4-associated cholangitis rarely occurs in the absence of pancreatitis, for which there was no evidence, and her IgG4 level was normal [10].

\section{Conclusions}

Both scleroderma and PSC are fibrotic diseases with widespread connective tissue disturbance occurring in CREST and abnormal collagen deposition in the bile duct epithelium. While PSC has been reported to occur in diffuse cutaneous systemic sclerosis, this is the first case report of PSC occurring with limited cutaneous systemic sclerosis.

\section{Consent}

Written informed consent was obtained from the patient for publication of this case report and any accompanying images. A copy of the written consent is available for review by the Editor-In-Chief of this journal.

\footnotetext{
Abbreviations

BSEP: bile salt export pump; CREST: calcinosis cutis, Raynaud phenomenon, esophageal dysmotility, sclerodactyly and telangiectasia; CT: computed tomography; ERCP: endoscopic retrograde cholangiopancreatography; IgG4: immunoglobulin G4; LFT: liver function tests; MRCP: magnetic resonance cholangiopancreatography; PBC: primary biliary cirrhosis; PSC: primary sclerosing cholangitis.
}

\section{Competing interests}

The authors declare that they have no competing interests.

\section{Authors' contributions}

AP was the medical registrar as part of the medical team caring for this patient and clinically assessed her as well as organizing and reviewing relevant investigations. She did a literature review and wrote the case report. JM was the consultant rheumatologist attending this patient as both an in-patient and out-patient. He read this case report and made several amendments and suggestions. Both authors read and approved the final manuscript.

\section{Acknowledgements}

We thank Professor lan Roberts-Thomson, gastroenterologist, for his review of this case report.

\author{
Author details \\ ${ }^{1}$ The Queen Elizabeth Hospital, Adelaide, SA, Australia. ${ }^{2}$ University \\ Department of Medicine, Modbury Hospital, Adelaide, SA, Australia.
}

Received: 3 May 2015 Accepted: 26 October 2015

Published online: 25 November 2015

\section{References}

1. Chapman R, Fevery J, Kalloo A, Nagorney DM, Boberg KM, Shneider B, et al. Primary sclerosing cholangitis. Hepatology. 2010;51:660-78.

2. Fraile G, Rodriguez-Garcia JL, Moreno A. Primary sclerosing cholangitis associated with systemic sclerosis. Postgrad Med J. 1991;67:189-92.
3. Abraham S, Begum S, Isenberg D. Hepatic manifestations of autoimmune rheumatic diseases. Ann Rheum Dis. 2004;63:123-9.

4. Murray-Lyon IM, Thompson RPH, Ansell ID, Williams R. Scleroderma and primary biliary cirrhosis. BMJ. 1970;3:258-9.

5. Fattinger K, Funk C, Pantze M, Weber C, Reichen J, Stieger B, et al. The endothelin antagonist bosentan inhibits the canalicular bile salt export pump: A potential mechanism for hepatic adverse reactions. Clin Pharmacol Ther. 2001;69:223-31.

6. Treiber A, Aanismaa P, de Kanter R, Delahaye S, Treher M, Hess P, et al. Macitentan does not interfere with hepatic bile salt transport. J Pharmacol Exp Ther. 2014:350:130-43.

7. Yimam KK, Bowlus CL. Diagnosis and classification of primary sclerosing cholangitis. Autoimmun Rev. 2014;13:445-50.

8. Dave M, Elmunzer BJ, Dwamena BA, Higgins PD. Primary sclerosing cholangitis: a meta-analysis of diagnostic performance of MR cholangiopancreatography. Radiology. 2010;256:387.

9. Kumagi T, Heathcote EJ. Primary biliary cirrhosis. Orphanet J Rare Dis. 2008:3:1-17.

10. Chapman R, Fevery J, Kalloo A, Nagorney DM, Boberg KM, Shneider B, et al. Diagnosis and management of primary sclerosing cholangitis. Hepatology. 2010;51:660-78.

\section{Submit your next manuscript to BioMed Central and take full advantage of:}

- Convenient online submission

- Thorough peer review

- No space constraints or color figure charges

- Immediate publication on acceptance

- Inclusion in PubMed, CAS, Scopus and Google Scholar

- Research which is freely available for redistribution 